\title{
Déterminisme génétique de la résistance au puceron vert (Myzus persicae) chez le pêcher. Résultats complémentaires
}

\author{
R Monet ${ }^{*}$, G Massonié ${ }^{2}$ \\ ${ }^{1} / N R A$, centre de recherches de Bordeaux, station de recherches fruitières; \\ ${ }^{2}$ INRA, centre de recherches de Bordeaux, station de zoologie, BP 81, F33883 Villenave-d'Ornon cedex, France.
}

(Reçu le 28 octobre 1993; accepté le 17 janvier 1994)

\begin{abstract}
Résumé - Chez le pêcher, une étude génétique du mode d'hérédité de la résistance à Myzus persicae a été faite à partir d'un géniteur résistant $\$ 2678: 47$, et d'un géniteur sensible $\$ 1161: 12$. Cette résistance a un déterminisme génétique simple : elle est dominante et monogénique (symboles, $R m 1, r m 1$ ). II n'y a pas de liaison entre le locus qui détermine la résistance et les loci qui concernent le port pleureur $(P l$, $p)$, le triangle liégeux $(T, t)$, le type de fleur $(S h, s h)$ et la couleur de la chair du fruit $(Y$, $y)$. La résistance s'est maintenue sur 4 générations consécutives et n'a pas jusqu'ici été surmontée. Cette étude fait partie d'un programme de création de variétés résistantes à Myzus persicae.
\end{abstract}

\section{Prunus persica/puceron vert/génétique de la résistance/liaisons génétiques}

\begin{abstract}
Summary - Genetic determinism of resistance to the green aphid (Myzus persicae) in the peach. Further results. The mode of heredity of resistance to Myzus persicae was established in the peach using

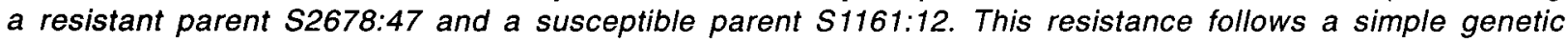
determinism: it is dominant and monogenic (Symbols Rm1, rm1). No link was found between the locus determining aphid resistance and the loci determining weeping shape $(\mathrm{Pl}, \mathrm{pl})$, corky triangle $(T, \mathrm{t})$, flower shape (Sh, sh), and fruit flesh colour $(\mathrm{Y}, \mathrm{y})$. Aphid resistance was maintained over 4 consecutive generations and has not been overcome yet. This study is part of a breeding programme to create varieties resistant to Myzus persicae.
\end{abstract}

Prunus persica/green aphid/genetics of resistance/genetic linkages

\section{INTRODUCTION}

Parmi les aphides présents sur le pêcher, le puceron vert, Myzus persicae (Sulzer) est le plus abondant et le plus difficile à combattre car très résistant aux insecticides de synthèse (Cravedi et Cervato, 1993). Par ailleurs, en dehors des dégâts directs qu'il provoque sur différents organes de l'arbre, il est aussi vecteur du virus de la Sharka. Cette maladie très grave chez le pêcher est en rapide expansion dans tous les pays d'Europe méridionale et ne cesse d'inquiéter les producteurs. On conçoit que la création de variétés résistantes au puceron vert devienne urgente car, si elle peut permettre de réduire les coûts de production et la pollution par les insecticides, elle pourrait éventuellement ralentir l'expansion de la Sharka en réduisant les populations de Myzus.

La création de variétés résistantes à un parasite nécessite de posséder des géniteurs de résistance et de connaître, lorsque celle-ci existe, son mode d'hérédité. Massonié et al

\footnotetext{
*Correspondance et tirés à part
} 
(1982) ont testé la résistance à Myzus persicae de nombreux clones à l'intérieur de l'espèce Prunus persica, chez les espèces voisines ( $P$ cerasifera, $P$ davidiana, $P$ kansuensis) et des hybrides interspécifiques. Ils ont pu caractériser ainsi quelques sources de résistance, notamment celle du clone de pêcher S2678 dont il sera question ci-dessous et de $P$ davidiana utilisé comme géniteur de résistance dans un programme d'hybridation interspécifique à la station de recherches fruitières méditerranéennes INRA d'Avignon (Sauge, 1992).

Le clone $\$ 2678$ est aussi intéressant par son port naturel pleureur qui peut être susceptible de faciliter la culture du pêcher à haute densité; ce géniteur avait été, dès 1971 , utilisé dans un programme de création de variétés à port pleureur à la station de recherches fruitière INRA de Bordeaux. II était donc possible de mener conjointement, à partir d'un même géniteur, un programme de création variétale mais aussi de résistance à Myzus persicae.

La première phase de ce travail a consisté à rechercher le déterminisme génétique de ces 2 caractères. Nous avons publié une étude complète (Monet et al, 1988) concernant le caractère port pleureur. Pour la résistance au puceron vert, nous avons indiqué dans une note préliminaire (Massonié et al, 1984) que le déterminisme génétique de la résistance était simple, dominant et monogénique.

Nous donnons ici un développement à cette note. Le déterminisme génétique de la résistance sera observé sur plusieurs générations ; et nous examinerons ses liaisons avec d'autres caractères mendéliens du pêcher qui étaient en disjonction dans ce croisement.

\section{MATÉRIEL ET MÉTHODES}

\section{Les géniteurs initiaux}

Trois cultivars ont été utilisés comme géniteurs primaires: Weeping Flower Peach, Redhaven et Early Sungrand.

Weeping Flower Peach (clone S2678) est un pêcher ornemental à fleurs doubles originaires des États-Unis. Il est aussi à port pleureur et résistant à Myzus persicae. Ses fruits n'ont pas de valeur marchande. Ce géniteur n'a pas été utilisé direc- tement en croisement ; ce sont 2 de ses descendants issus d'autofécondation qui ont été utilisés soit : S2678:47 et S2678:48. L'arbre S2678:48 qui figure dans l'étude génétique du caractère port pleureur (Monet et al, 1988) ne sera pas concerné ici, car il introduisait le caractère indésirable « fleur double".

Redhaven (clone S1161) était très cultivé au moment où le travail a débuté; il a été retenu comme géniteur améliorant. C'est une variété de pêches à chair jaune originaire des États-Unis sensible à Myzus persicae. Redhaven n'a pas été utilisé directement en croisement ; c'est son descendant pas autofécondation $\mathrm{S1161:12}$ qui a été choisi comme parent femelle.

Early Sungrand (clone S3955) apparaît en $3^{e}$ génération: il est utilisé comme géniteur améliorant. C'est une variété de nectarines à chair jaune provenant des États-Unis, sensible à Myzus persicae. Early Sungrand a été utilisé directement en croisement.

\section{Les étapes du programme}

Les différentes étapes de ce travail se sont échelonnées sur plus de 20 ans; elles sont résumées dans la figure 1.

Pour vérifier la pureté des caractères port pleureur et résistance à Myzus persicae chez les géniteurs Weeping Flower Peach et Redhaven, nous les avons, dès le départ, soumis à autofécondation. Ceci permettait aussi d'établir leur formule génotypique pour les caractères mendéliens qui étaient concernés par cette étude et avoir une idée de leur valeur en tant que géniteurs.

Dans la famille d'autofécondation de Redhaven et dans celle de Weeping Flower Peach, nous avons retenu les arbres $\$ 1161: 12$ et $\$ 2678: 47$ qui ont été hybridés pour constituer la famille $\mathrm{F} 1$ de l'étude génétique soit $S(1161: 12 \times 2678: 47)$. Ce croisement n'a donné que 2 arbres.

Pour constituer la génération F2 de l'étude génétique, nous avons soumis à autofécondation le premier d'entre eux qui a donné la famille $S(1161: 12 \times 2678: 47) 1$ : de 55 arbres.

Dans cette génération F2, 2 arbres ont été sélectionnés, l'un à port pleureur $\mathrm{S}(1161: 12 \times$ $678: 47) 1: 35$ et l'autre à port normal $\mathrm{S}(1161: 12 \times 2678: 47) 1: 55$. Leurs aptitudes agronomiques les plaçaient en tête de la famille; ils étaient par ailleurs résistants à Myzus persicae. Ces 2 géniteurs ont été croisés avec Early Sungrand $\mathrm{S} 3655$ pour créer la $3^{\mathrm{e}}$ génération. Simultanément nous avons autofécondé l'arbre $S(1161: 12 \times 2678: 47) 1: 55$ pour vérifier son état hétérozygote pour le caractère port pleureur. 


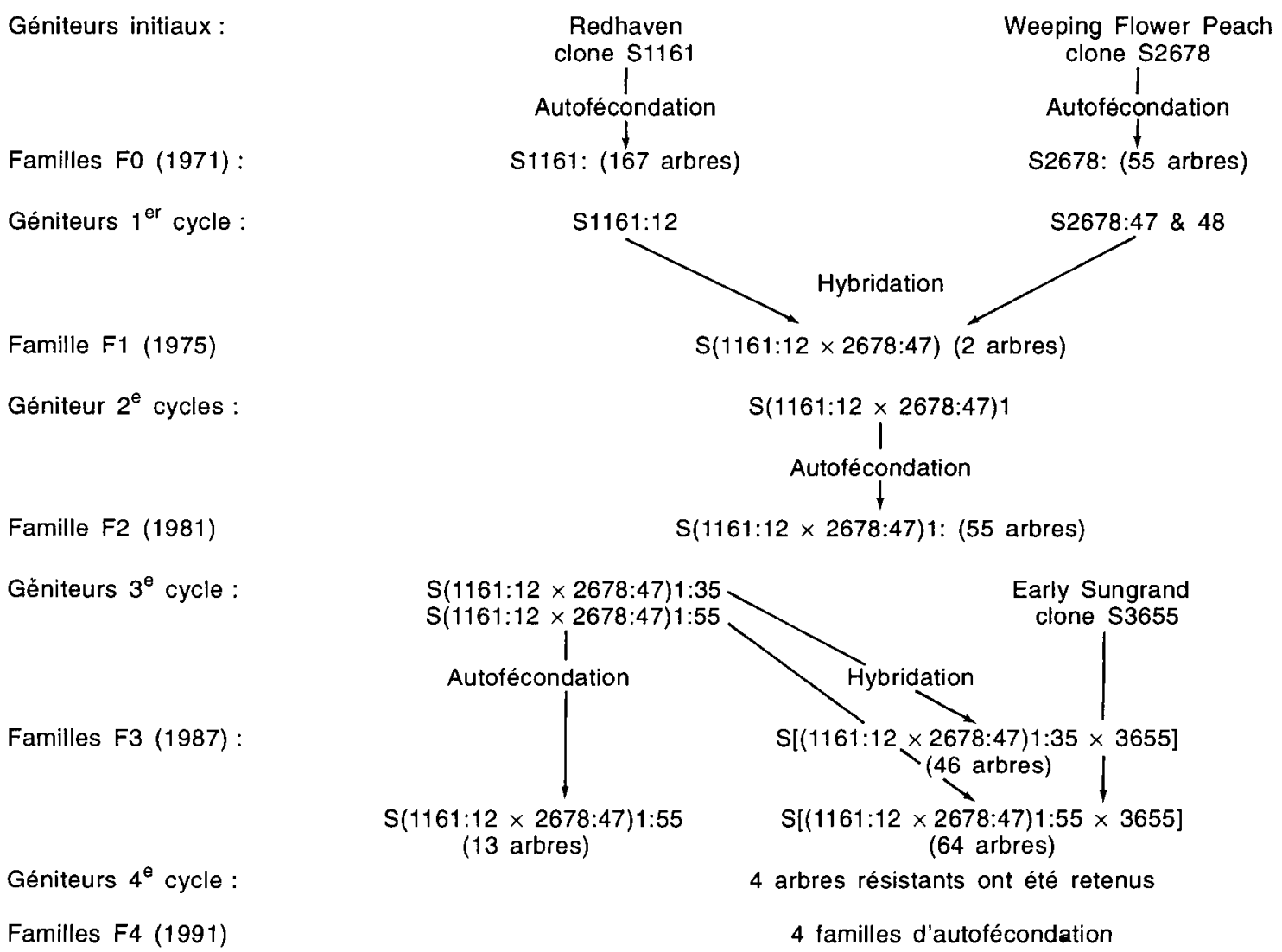

Fig 1. Étapes du programme d'étude de la résistance et de l'amélioration pour la résistance à Myzus persicae.

Dans la $3^{\mathrm{e}}$ génération, nous avons choisi, uniquement dans la famille $S[(1161: 12 \times 2678: 47)$ $1: 55 \times 3655$ ], différents géniteurs qui ont été soumis à autofécondation pour fournir la $4^{\mathrm{e}}$ génération. Nous ne parlerons pas de cette dernière, qui concerne beaucoup plus l'aspect amélioration que l'aspect étude génétique.

\section{Mise en évidence de la résistance au puceron}

Pour tester la résistance ou la sensibilité d'un arbre au puceron vert, nous intervenons au mois de mai. Nous fixons, sur une jeune pousse en pleine croissance de l'arbre à tester, une pousse portant plusieurs colonies de fondatrigènes de Myzus persicae prélevée sur un arbre sensible; chaque fondatrigène donnant une lignée, plusieurs lignées de pucerons sont donc utilisées. Si l'arbre est sensible, il y a installation des pucerons sur la pousse testée. Si l'arbre est résistant, il n'y a pas colonisation mais les piqûres des pucerons provoquent l'apparition de ponctuations anthocyanées ou jaunes pouvant évoluer en nécroses. Les pucerons se dispersent puis finissent par quitter l'arbre après avoir effectué de nouvelles tentatives d'installation sur d'autres pousses. Un $2^{\mathrm{e}}$ test est réalisé l'année suivante pour confirmer la première observation.

\section{RÉSULTATS}

\section{Comportement héréditaire de la résistance au puceron}

Weeping Flower Peach (S2678) a donné, par autofécondation, une famille de 55 arbres, tous résistants à Myzus.

Redhaven (S1161), clone sensible, a donné une famille de 167 arbres dans laquelle nous n'avons testé qu'un échantillon de 10 arbres qui étaient tous sensibles et notamment l'arbre S1161:12.

Les 2 géniteurs initiaux et leurs descendants par autofécondation étaient donc homozygotes pour les caractères de résistance et de sensibilité. 
Tableau I. Répartition des individus résistants et sensibles à Myzus persicae dans 2 familles issues d'autofécondation et une famille obtenue par hybridation.

\begin{tabular}{lccccc}
\hline \multicolumn{1}{c}{ Familles } & Résistants & Sensibles & $\begin{array}{c}\text { Prop } \\
\text { théo }\end{array}$ & $\chi_{\text {ob }}^{2}$ & Signif \\
\hline $\mathrm{S}(1161: 12 \times 2678: 47) 1:$ & $42(41,25)$ & $13(13,75)$ & $3: 1$ & 0,05 & NS \\
$\mathrm{S}(1161: 12 \times 2678: 47) 1: 55:$ & $9(9,75)$ & $4(3,25)$ & $3: 1$ & 0,23 & $\mathrm{NS}$ \\
$\mathrm{S}[(1161: 12 \times 2678: 47) 1: 55 \times 3655]$ & $17(15,5)$ & $14(15,5)$ & $1: 1$ & 0,29 & NS \\
\hline
\end{tabular}

Les chiffres entre parenthèses sont les valeurs théoriques correspondant aux proportions de la colonne 4. NS: non significatif au seuil $5 \%$ par le test de $\chi^{2}\left(\chi_{\text {th }}^{2}=3,84\right.$ pour 1 degré de liberté).

Les 2 arbres de la génération $\mathrm{F} 1$ issus du croisement $\quad$ S1161:12 $\times$ S2678:47 étaient résistants. L'effectif de 2 arbres n'est pas suffisant pour parler de dominance du caractère de résistance ; cependant l'étude ultérieure d'autres familles confirme ce fait (tableau I).

Ainsi dans la famille $\mathrm{F} 2$ issue de l'autofécondation de l'arbre résistant $S(1161: 12 \times$ 2678:47)1, nous avons observé une disjonction en accord avec les proportions mendéliennes de 3 résistants pour 1 sensible (tableau I).

Dans la famille issue de l'autofécondation de l'arbre résistant $S(1161: 12 \times 2678: 47) 1: 55$, nous avons obtenu une disjonction compatible avec une ségrégation de type 3:1 avec 9 individus résistants pour 4 sensibles. L'arbre était donc hétérozygote pour le caractère de résistance (tableau I).

Enfin dans la famille issue du croisement de l'arbre hétérozygote $S(1161: 12 \times 2678: 47)$ 1:55 par Early Sungrand S3655, cultivar sensible, nous avons testé les 31 premiers arbres de la famille et obtenu une disjonction de type 1:1 (tableau I).

La résistance s'est comportée dans ces différentes familles comme dominante et monogénique. Nous proposons les symboles $R m 1$ et $r m 1$ pour dénommer les allèles de résistance et de sensibilité au puceron vert.

\section{Liaisons génétiques entre le caractère de résistance et d'autres caractères mendéliens en disjonction dans la génération F2}

Les familles issues de l'autofécondation de Weeping Flower Peach et Redhaven sont homogènes pour les caractères qui font l'objet de l'étude génétique; de ce fait chaque indi- vidu de ces familles est homozygote pour ces caractères.

Le géniteur S2678:47 issu de l'autofécondation de Weeping Flower Peach, est ainsi homozygote pour les caractères suivants:

- résistance à Myzus, génotype : $R m 1, R m 1$;

- port pleureur, génotype : $p l, p l$;

- triangle liégeux absent, génotype: $T, T$;

- fleur rosacée, génotype: sh, sh;

- chair blanche, génotype: Y, Y.

Le géniteur $S 1161: 12$ issu de l'autofécondation de Redhaven est, pour la même raison, homozygote pour les caractères :

- sensibilité à Myzus, génotype : rm1, rm1;

- port normal, génotype : $P I, P l$;

- triangle liégeux présent, génotype : $t, t$;

- fleur campanulée, génotype : Sh, Sh;

- chair jaune, génotype : $Y, Y$.

Les hybrides $\mathrm{F} 1$ sont donc hétérozygotes pour ces 5 caractères et la génération $F 2$ doit permettre l'étude des liaisons génétiques éventuelles pouvant exister entre eux.

Le tableau II présente les résultats obtenus pour les couples de caractères: résistance/port de l'arbre, résistance/triangle liégeux, résistance/type de fleur, résistance/couleur de la chair du fruit. Dans chaque cas la répartition des 4 phénotypes possibles n'est pas statistiquement différente des effectifs théoriques d'une disjonction 9:3:3:1 caractérisant l'indépendance génétique des caractères étudiés.

Les associations de caractères (et leurs formes alléliques) : port de l'arbre/triangle liégeux, port de l'arbre/type de fleur, port de l'arbre/couleur de la chair du fruit, triangle liégeux/type de fleur, triangle liégeux/couleur de la chair du fruit et type de fleur/couleur de la chair du fruit, ont déjà été étudiées (Monet et al, 1988). Toutes ces associations donnent des disjonctions indépendantes, excepté le couple : triangle liégeux/type de fleur, pour lequel il existe une liaison génétique faible. 
Tableau II. Répartition des phénotypes dans la descendance par autofécondation du S(1161:12 $\times 2678: 47) 1$.

\begin{tabular}{|c|c|c|c|}
\hline Phénotypes & Résistants & Sensibles & Total \\
\hline \multicolumn{4}{|c|}{ Pour les caractères de résistance/port de l'arbre } \\
\hline $\begin{array}{l}\text { Port normal } \\
\text { Port pleureur } \\
\text { Total }\end{array}$ & $\begin{array}{r}34(30,94) \\
8(10,30) \\
42(41,25)\end{array}$ & $\begin{array}{r}11(10,30) \\
2(3,44) \\
13(13,75)\end{array}$ & $\begin{array}{l}45(41,25) \\
10(13,75) \\
55\end{array}$ \\
\hline \multicolumn{4}{|c|}{ Pour les caractères de résistance/triangle liégeux } \\
\hline $\begin{array}{l}\text { Sans triangle } \\
\text { Avec triangle } \\
\text { Total }\end{array}$ & $\begin{array}{l}30(30,94) \\
12(10,30) \\
42(41,25)\end{array}$ & $\begin{array}{r}10(10,30) \\
3(3,44) \\
13(13,75)\end{array}$ & $\begin{array}{l}40(41,25) \\
15(13,75) \\
55\end{array}$ \\
\hline \multicolumn{4}{|c|}{ Pour les caractères de résistance/type de fleurs } \\
\hline $\begin{array}{l}\text { Campanulée } \\
\text { Rosacée } \\
\text { Total }\end{array}$ & $\begin{array}{r}33(30,94) \\
9(10,30) \\
42(41,25)\end{array}$ & $\begin{array}{r}10(10,30) \\
3(3,44) \\
13(13,75)\end{array}$ & $\begin{array}{l}43(41,25) \\
12(13,75) \\
55\end{array}$ \\
\hline \multicolumn{4}{|c|}{ Pour les caractères de résistance/couleur de la chair du fruit } \\
\hline $\begin{array}{l}\text { Chair blanche } \\
\text { Chair jaune } \\
\text { Total }\end{array}$ & $\begin{array}{r}35(30,94) \\
7(10,30) \\
42(41,25)\end{array}$ & $\begin{array}{r}10(10,30) \\
3(3,44) \\
13(13,75)\end{array}$ & $\begin{array}{l}45(41,25) \\
10(13,75) \\
55\end{array}$ \\
\hline
\end{tabular}

Les chiffres entre parenthèses sont ceux d'une disjonction théorique 3:1 (fréquences marginales), et 9:3:3:1 (fréquences des caractères associés 2 à 2).

\section{DISCUSSION}

Bien que l'appréciation de la résistance ne soit pas toujours aisée sur le terrain (la colonisation par le puceron peut être entravée par des conditions climatiques défavorables et sans doute par l'état nutritionnel de l'arbre), on constate qu'elle s'est maintenue au cours du temps, et que, d'une génération à l'autre, son déterminisme reste monogénique et dominant. Ce déterminisme simple facilite la gestion d'un programme de création de variétés résistantes au puceron vert du pêcher.

Nous n'avons pas trouvé de liaison entre le locus du gène de résistance et les loci déterminant le port de l'arbre, la présence ou l'absence de triangle liégeux, le type de fleur et la couleur de la chair du fruit. L'effectif relativement faible des familles rend cependant difficile la mise en évidence de liaisons génétiques supérieures à 40 centimorgans ; une disjonction apparemment indépendante peut donc masquer quelquefois une liaison lâche.

En ce qui concerne les étapes du programme d'amélioration, il faut noter le goulot génétique que constitue la génération $\mathrm{F} 1$ qui ne comprenait que 2 arbres. S'il n'a pas été un obstacle à la transmission de la résistance, il a pu ralentir le progrès vers l'amélioration de la qualité du fruit. Cette progression a été lente; la $5^{\ominus}$ génération (famille F4) qui sera observée pour la première fois en 1993, devrait permettre d'isoler les premières présélections. On voit ici le temps nécessaire à introduire une résistance chez une espèce fruitière.

\section{CONCLUSION}

Les études génétiques conduites chez les arbres fruitiers restent toujours difficiles car il faut travailler sur des familles à effectif réduit obtenues à partir de géniteurs dont on ne connaît qu'imparfaitement la formule génotypique. Une étude génétique ne se justifie que si elle est le support d'un travail d'amélioration; elle est d'ailleurs le plus souvent le sous-produit de celui-ci. Nous nous sommes cependant efforcés, dans ce travail, de suivre les règles habituelles d'une étude génétique mendélienne classique : vérification de la pureté des géniteurs pour les caractères dont 
on veut établir l'hérédité, contrôle strict des hybridations et autofécondations, pas de sélection précoce qui pourrait altérer les fréquences phénotypiques. II faut considérer toutefois qu'un résultat n'est définitivement acquis que s'il est confirmé dans d'autres familles.

Cette résistance est-elle susceptible d'être contournée? II a déjà été observé pour Myzus persicae l'apparition de souches résistances aux insecticides (Cravedi et Cervato, 1993). Pour ce qui est de résistances génétiques, celle qui vient d'être étudiée est la première qui a été mise en évidence chez le pêcher. Dans les conditions de notre travail, c'est-àdire sur un effectif d'arbres assez faible, la résistance s'est maintenue sur une longue durée. II faudra que la culture d'arbres portant le gène de résistance se développe pour que l'on puisse apprécier sa réelle valeur.

\section{RÉFÉRENCES}

Cravedi P, Cervato P (1993) Researches on Myzus persicae (Sulz) résistance to insecticides. OILB/SROP 4, 1-3

Massonié G, Maison P, Monet R, Grasselly C (1982) Résistance au puceron vert du pêcher, Myzus persicae Sulzer (Homoptera Aphididae) chez Prunus persicae (L) Batsch et d'autres espèces de Prunus. agronomie 2 63-70

Massonié G, Monet R, Bastard Y, Maison P (1984) Heredity in peach of the hypersensitivity reaction to the green peach aphid Myzus persicae Sulzer. OILB/SROP 7, 69

Monet R, Bastard Y, Gibault B (1988) Étude génétique du caractère «port pleureur» chez le pêcher. agronomie 8 353-358

Sauge MH (1992) Sélection pour la résistance au puceron vert (Myzus persicae Sulzer) chez le pêcher (Prunus persica L Batsch), mémoire ENSFA, Rennes, $36 \mathrm{p}$ 